\title{
Ventral Hernia
}

National Cancer Institute

\section{Source}

National Cancer Institute. Ventral Hernia. NCI Thesaurus. Code C118313.

The protrusion of abdominal cavity contents through the anterior abdominal wall. 